${ }^{1}$ F. Zwicky, Proc. Nat. Acad. Sci., 15, 816 (1929); Helvetia Physica Acta, 3, 269 (1930) and 4, p. 49 (1931).

2 E. Berger, Forschungen und Fortschritte, p. 184 (1931).

${ }^{3}$ F. M. Jaeger and E. Rosenbohm, Proc. Amsterdam Acad. Sci., 33, No. 5, 1930, and 34, No. 1, 1931.

${ }^{4}$ K. F. Herzfeld, Phys. Rev., 29, 701 (1927).

${ }^{5}$ F. Bitter, Ibid., 91 (1931).

6 U. Dehlinger, Ann. Physik, 2, 786, 1929.

\title{
ON THE AIR RESISTANCE OF PROJECTILES
}

\author{
By Paul S. Epstein
}

California Institute of Technology

Communičated July 3, 1931

1. Introductory.-In making this study, the author had in mind its applications to the problem of meteoric impact. The different laws of resistance, heretofore proposed, contain too many empirical or hypothetical elements to permit a safe extrapolation to cosmic velocities. A more thorough investigation into the theoretical aspects of the problem seemed, therefore, desirable. It is known from the experimental results of aeronautics and hydraulics and from their theoretical analysis, that, for moderate velocities, already, the influence of viscosity is small compared with that of inertia. It seems, therefore, permissible to neglect the viscous terms altogether even in the range of velocities attained by ordinary projectiles. The problem is reduced, by this remark, to finding solutions of Euler's equations of hydrodynamics for velocities higher than the velocity of sound. While looking about for a simple case in which the solution could be carried through, it occurred to the author that in the two-dimensional case certain well-known integrals of Euler's equations due to Riemann, ${ }^{1}$ Prandtl $^{2}$ and Th. Meyer $^{3}$ permit a rigorous solution of the problem for polygonal contours. For simplicity, we take a quadrangle symmetrical with respect to one of its diagonals and moving in the direction of this diagonal (Fig. 1). We shall find the rigorous expression for the resistance of such a body in section 7 . For lower velocities the formulae are rather involved, but for the limit of very high velocities they are reduced to the very simple expression (28) which remains valid for contours with curved sides and for three dimensional bodies. The properties of our solution and its relation to some laws of ballistic resistance proposed by others and to experiment are discussed in section 8 .

2. Some Mathematical Aspects of the Problem.-The case of high velocity is in some respects simpler than that of low velocity. The front point 
$A$ of the projectile is moving with a velocity higher than the velocity of sound. Therefore, the air to the left side of it has no intimation of the projectile's approach and remains at rest up to the very point $A$. The line separating the region of resting air from that of moving air is obtained by a simple reasoning due to Mach. ${ }^{4}$ At every moment $(t=0)$ the point $A$ can be regarded as the source of a spherical wave. After the lapse of time $t$, this spherical wave will have expanded to the radius $R=a t$, where $a$ is the velocity of the wave front under the particular conditions of the problem. In the same time the point $A$ will have progressed to a new position $A^{\prime}$ through the distance $l=w t$. It is easy to see that a straight line, going out from the point $A^{\prime}$ and tangent to the circle of radius $R$ with its center in $A$, is the envelope of all circular waves starting from the front point in its different positions. ${ }^{5}$ This line is, therefore, the line of discontinuity separating the two regions of resting and of moving air.

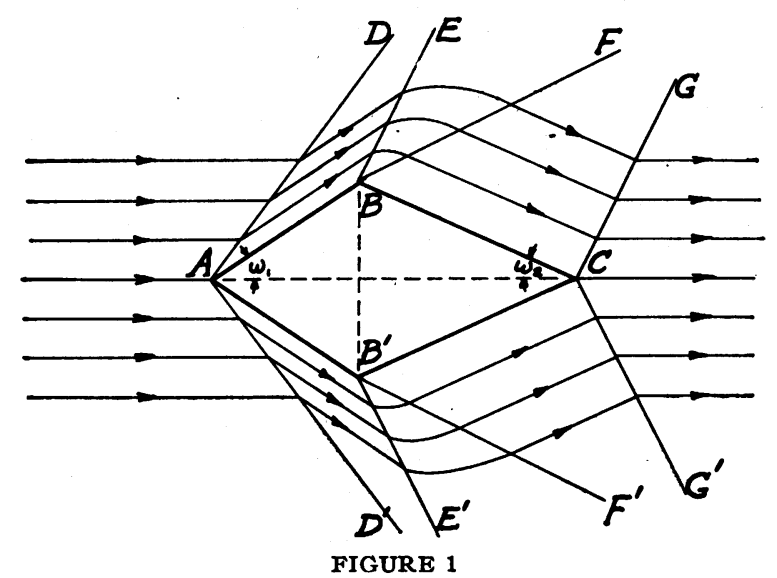

Its angle of inclination $\alpha$ to the direction of motion is given by the simple relation $\sin \alpha=R / l$ or

$$
\sin \alpha=a / w
$$

and is called Mach's angle.

Quite similar conditions obtain in the case of a current streaming along a plane wall. If the velocity of this current is higher than that of sound, any sudden break in the wall, or other irregularity, cannot produce any effect on the character of motion in a region limited by a straight line going out from this irregularity and including Mach's angle with the current. These conditions have the consequence that the air filled space around our polygon (Fig. 1) breaks up into a number of sections separated by straight lines of discontinuity. The coinplete solution can then be pieced together from two simple solutions given by Riemann and by Prandtl. We give these two solutions in the sections 3,4 and 5 . 
3. Riemann's Shock Wave.-It was shown by Riemann that in a compressible medium there can exist surfaces of discontinuity moving with a certain velocity in the direction of their own normal. Let us regard a plane discontinuity or "shock wave" from the point of view of the observer moving with it, so that the shock wave will appear to us stationary. We denote pressure, density and normal velocity of the medium, to one side of the discontinuity, by $p_{1}, \rho_{1}, v_{1}$, to the other side of it, by $p_{2}, \rho_{2}, v_{2}$. It will be convenient to regard the discontinuity as the limiting case of a very thin layer in which the corresponding quantities are denoted by $p, \rho, v$ and in which they change continuously from $p_{1}, \rho_{1}, v_{1}$ to $p_{2}, \rho_{2}, v_{2}$. Let us further denote the total velocity by $w$ and the component parallel to the inhomogeneous layer by $u$. We start from Euler's equations for a two dimensional stationary electrodynamical field:

$$
\left.\begin{array}{c}
\left.\rho\left(u \frac{\partial u}{\partial x}+v \frac{\partial u}{\partial y}\right)=-\frac{\partial p}{\partial x} \cdot \rho\left(u \frac{\partial v}{\partial x}+v \frac{\partial v}{\partial y}\right)=-\frac{\partial p}{\partial y} \cdot\right) \\
\nabla \cdot(\rho \bar{v})=\frac{\partial(\rho u)}{\partial x}+\frac{\partial(\rho v)}{\partial y}=0 .
\end{array}\right\}
$$

We are interested only in irrotational solutions of these equations

$$
\vec{w}=-\nabla \psi \text {. }
$$

Owing to this form of $\bar{w}$, there exists the relation $\partial u / \partial y=\partial v / \partial x$, and the first two equations can be combined into the well-known relation of Bernoulli

$$
\frac{1}{2} d w^{2}+\frac{d p}{\rho}=0 .
$$

Let $y$ be the direction normal to the inhomogeneous layer. Then $\rho$ is independent of $x$ while $u$ is constant: the third eq. is, therefore, reduced to $\partial(\rho v) / \partial y=0$ or

$$
\rho v=\text { const. }=\rho_{1} v_{1}=\rho_{2} v_{2},
$$

while eq. (4) becomes $\frac{1}{2} d v^{2}+d p / \rho=0$. Substituting $v$ from eq. (5). we obtain

$$
d p=\rho_{1}^{2} v_{1}^{2} d \rho / \rho^{2} .
$$

The integral of this relation taken across the layer gives

$$
p_{2}-p_{1}=v_{1}^{2}\left(\rho_{2}-\rho_{1}\right) \rho_{1} / \rho_{2} \text {. }
$$

This is the fundamental equation of Riemann's shock wave giving the difference of pressure on both sides of the discontinuity in terms of the densities. 
We are going to apply these relations to the motion in a concave angle formed by two plane walls (Fig. 2). The shock wave separates two regions of the medium characterized by $\rho_{1}, p_{1}$ and $\rho_{2}, p_{2}$, respectively. The total velocities $w_{1}$ and $w_{2}$ are parallel to the walls $B A$ and $A C$. The components normal to the shock wave $v_{1}, v_{2}$ are subject to conditions (5), (7), while the tangential component $u$ is continuous and has the same value on both sides. We have the relations

$$
\left\{\begin{array}{ll}
u=w_{1} \cos \alpha, & v_{1}=w_{1} \sin \alpha, \\
u=w_{2} \cos (\alpha-\omega), & v_{2}=w_{2} \sin (\alpha-\omega) .
\end{array}\right\}
$$

We can eliminate $w_{1}$ and $w_{2}$ from these formulae. If we take into account equation (5) and denote, for short, $\rho_{2} / \rho_{1}=x$, the result of this elimination is $\operatorname{tg} \alpha=x \operatorname{tg}(\alpha-\omega)$ or

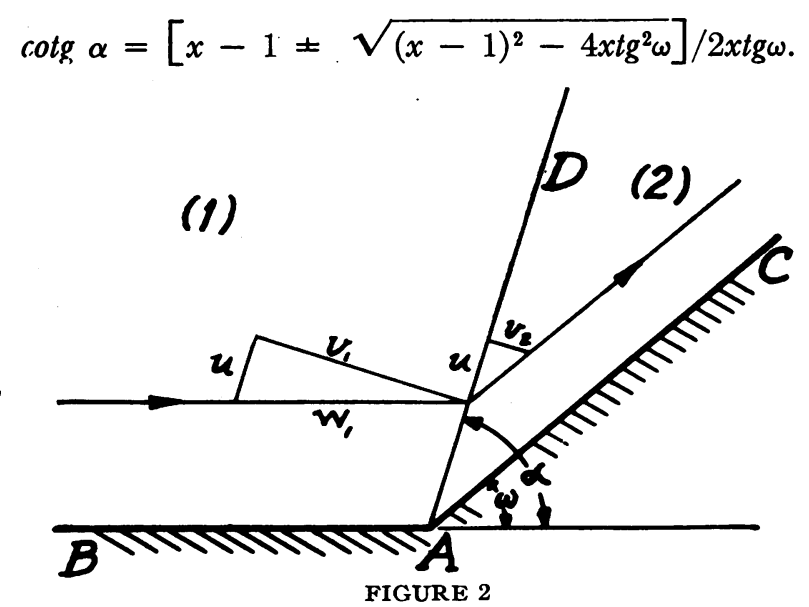

This equation gives us the angle of inclination $\alpha$ of the shock wave as a function of the condensation $x$. It is interesting to note that there are two possible solutions of the problem, corresponding to the two signs of the square root. There is no mathematical reason to prefer one solution to the other, but there is a dynamical one which rules out the minus sign. We shall, therefore, omit this sign in the following formulae and we shall return to the physical discussion of our reasons for this in section 8 . On the other hand equation (7) can be thrown into the form

$$
\frac{p_{2}-p_{1}}{\rho_{2}-\rho_{1}}=\frac{\rho_{1}}{\rho_{2}} w_{1}^{2} \sin ^{2} \alpha
$$

Substituting $\alpha$ from the preceding equation, we obtain

$$
\frac{\rho_{1} w_{1}^{2}}{p_{1}}=\left[\frac{x-1+\sqrt{(x-1)^{2}-4 x \operatorname{tg}^{2} \omega}}{2 x \operatorname{tg}^{2} \omega}+1\right] \cdot\left(\frac{p_{2}}{p_{1}}-1\right),
$$


a formula giving the relation between the original velocity $w_{1}$ and the condensation $x$, if the law connecting pressure and density

is known.

$$
p=f(\rho)
$$

4. Pressure-Density Relation in a Shock Wave.-There seems to exist little clarity as to the form which equation (11) has in a shock wave. Most authors have used the adiabatic relation $p=k \rho^{\gamma}$, however, it is obvious that the conditions of compression in a shock wave are vastly different from those in a stationary vessel, so that there is not much justification for this law. Let us set up the equation of the energy balance for the shock wave. We follow a unit of mass of air in its motion across the discontinuity and assume that the total energy change in it is equal to nothing.

$$
U_{2}-U_{1}+\frac{1}{2}\left(w_{2}^{2}-w_{1}^{2}\right)+W=0 .
$$

We denote by $U$ the internal energy of the gas, by $W$ the work which it does in moving from one side of the discontinuity to the other. This work consists of two items: on the one hand, it must push away a volume of gas equal to its own $\left(V_{2}=1 / \rho_{2}\right)$ on the front side, on the other hand, it vacates the volume $V_{1}=1 / \rho_{1}$, on the back side, allowing the gas behind it to do work against it. The balance is, obviously,

$$
W=p_{2} V_{2}-p_{1} V_{1}=\rho^{2} p d V+\rho^{2} V d p
$$

The increase of kinetic energy is known from equation (4) to be $\frac{1}{2} d w^{2}+$ $V d p=0$, which gives

$$
\frac{1}{2}\left(w_{2}^{2}-w_{1}^{2}\right)+\int_{1}^{2} V d p=0 .
$$

Subtracting it from (12), we find

$$
U_{2}-U_{1}+\int_{1}^{2} p d V=0
$$

This equation is satisfied by the adiabatic law of compression, and this may have been the reason why this law was used by many writers on the subject. However, there is an infinity of other laws satisfying the same relation and the adiabatic hypothesis leads to grave contradictions with the rest of our formulas. To find the true law we have only to replace the discontinuity by an inhomogeneous layer as in section 3 . The dependence of $p$ on $\rho$ is given there in equation (6) which we shall write in its integrated form

$$
p=p_{1}+\rho_{1}^{2} v_{1}^{2}\left(\frac{1}{\rho_{1}}-\frac{1}{\rho}\right) .
$$


Supposing that the equation of state of our gas is sufficiently well represented by $p V=R T$, and the internal energy by $U=C_{v} T=$ $p V /(\gamma-1)$, we find from (12), $\left(12^{\prime}\right)$ and (5)

$$
2 \gamma p_{1}+\left[\gamma-1+\frac{\gamma+1}{x}\right] \rho_{1} v_{1}^{2}=0 .
$$

Eliminating $v_{1}$ between this equation and (7), we arrive at the final law

$$
p_{2}=\frac{(\gamma+1) x-(\gamma-1)}{(\gamma+1)-(\gamma-1) x} p_{1} .
$$

This leads to the following expression for the change of internal energy

$$
U_{2}-U_{1}=C_{v}\left(T_{2}-T_{1}\right)=\frac{x^{2}-1}{[\gamma+1-(\gamma-1) x] x} \frac{p_{1}}{\rho_{1}} .
$$

It is easy to check that expressions (14) and (16) really satisfy the general condition (13). The law (15) is quite different from the adiabatic law $p_{2}=x^{\gamma} p_{1}$, and this means that inside the inhomogeneous layer, while the compression is going on, there is conduction of heat from element to element of the gas. However, every element loses as much energy in the second half of its path through this layer as it gains in the first half because, without this, a stationary flow could not be maintained. It is assumed that there is no appreciable loss of energy, by conduction and radiation, to bodies outside the wave. This assumption is sound enough as long as the compressions and temperature differences are not too high. We are going to discuss its limitations and the influence of possible deviations from it in section 9 . For the present, however, we shall base ourselves on this assumption and on formula (15) which is its mathematical expression. Substituting into $\left(10^{\prime}\right)$, we obtain our final formula

$\frac{w_{1}^{2}}{a_{1}^{2}}=\left[\frac{x-1+\sqrt{(x-1)^{2}-4 x \operatorname{tg}^{2} \omega}}{2 x \operatorname{tg}^{2} \omega}+1\right] \cdot \frac{2(x-1)}{(\gamma+1)-(\gamma-1) x}$

where $a^{2}=\gamma p / \rho$ is the square of the velocity of sound. This relation establishes the connection between the original velocity $w_{1}$ and the condensation $x$. As an immediate consequence of it, we can obtain the pressure $p_{2}$ by means of equation (15). If we wish to find $w_{2}$, we have to compute $\alpha$ from equation (9) and to use the relation $w_{2}=$ $w_{1} \cos \alpha / \cos (\alpha-\omega)$ which follows from equation (4).

5. The Prandtl-Meyer Solution.-This solution was published in Th. Meyer's thesis which is, at present, almost inaccessible. It is, therefore, well to give here a detailed exposition of it. Meyer's own derivation is somewhat cumbersome, and we present it here in a simplified form. 
We start from Euler's equations in two dimensions in the form (2), (3), (4) of section 3 . In polar coördinates the equations of energy and of continuity acquire the form

$$
\begin{gathered}
\frac{\rho}{2} d\left[\left(\frac{\partial \psi}{\partial r}\right)^{2}+\frac{1}{r^{2}}\left(\frac{\partial \psi}{\partial \varphi}\right)^{2}\right]+d p=0, \\
\frac{\partial}{\partial r}\left(r \rho \frac{\partial \psi}{\partial r}\right)+\frac{1}{r} \frac{\partial}{\partial \varphi}\left(\rho \frac{\partial \psi}{\partial \varphi}\right)=0 .
\end{gathered}
$$

We restrict our problem further by looking only for states of motion, in which the velocity $\bar{w}$ and the density $\rho$ are independent of $r$ and are functions of the angle $\varphi$ only. This will be the case when the potential has the form

$$
\psi=r \chi(\varphi)
$$

leading to the formulae

$$
\left.\begin{array}{c}
\frac{\rho}{2} d\left[\chi^{2}+\left(\frac{d \chi}{d \varphi}\right)^{2}\right]+d p=0, \\
\rho \chi+\frac{d}{d \varphi}\left(\rho \frac{d \chi}{d \varphi}\right)=0 .
\end{array}\right\}
$$

Multiplying the last equation by $d \chi$ we obtain

$$
\frac{\rho}{2} d\left[\chi^{2}+\left(\frac{d \chi}{d \varphi}\right)^{2}\right]+\left(\frac{d \chi}{d \varphi}\right)^{2} d \rho=0 .
$$

Comparing this with equation (18) we find

$$
v_{\varphi}^{2}=\left(\frac{d \chi}{d \varphi}\right)^{2}=\frac{d p}{d \rho}=f^{\prime}(\rho)=a^{2} .
$$

The azimutal component $v_{\varphi}$ of the velocity is always equal to the velocity of sound. This shows, among other things, that Prandtl's solution applies only to velocities higher than that of sound. Substituting (20) into (19), we obtain a relation between $\chi$ and $\rho$

$$
\chi^{2}+f^{\prime}(\rho)+2 \int \frac{f^{\prime}(\rho)}{\rho} d \rho=C .
$$

Differentiating this with respect to $\varphi$ and remembering that $d \chi / d \varphi=$ $\sqrt{f^{\prime}(\rho)}$, we arrive at a plain differential equation for $\rho$. There are no objections against the adiabatic law $f(\rho)=k \rho^{\gamma}$ in this case: in the absence of conduction and radiation, it is the correct connection between pressure 
and density. Assuming this law, the equation can be readily integrated, giving

$$
\left.\begin{array}{c}
\psi=r \sqrt{C} \sin \sqrt{\frac{\gamma-1}{\gamma+1}}\left(\varphi-\varphi_{0}\right), \\
{\left[\sqrt{\frac{C(\gamma-1)}{k \gamma(\gamma+1)}} \cos \sqrt{\frac{\gamma-1}{\gamma+1}}\left(\varphi-\varphi_{0}\right)\right] \frac{2}{\gamma-1} .}
\end{array}\right\}
$$

6. Applications.-We are now in a position to solve the problem of the flow around a corner (Fig. 3 ). Let the initial motion have the velocity $w_{2}$ parallel to the plane $A O$. According to our discussion in section 2, this type of motion will extend until the plane $O B$ going out from $O$ under Mach's angle:

$$
\sin \beta_{1}=a_{2} / w_{2},
$$

so that the angle $\beta_{1}$ can be considered as known. Beyond $O B$ the type

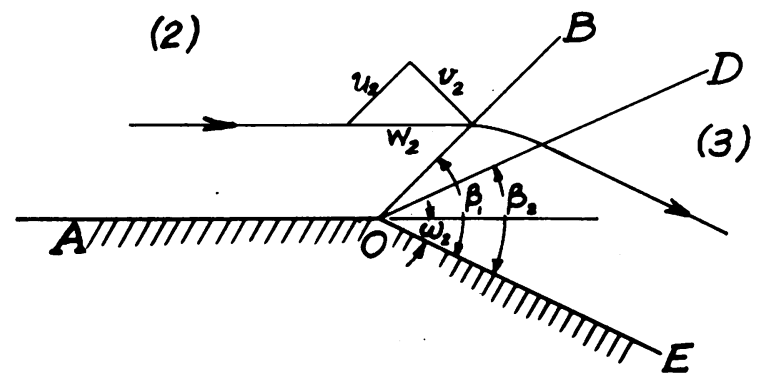

FIGURE 3

of motion will be that of Prandtl's solution (21). The two parameter's $C$ and $\varphi_{0}$ must be chosen so as to establish the continuity of the velocity across the line $O B$. If we call the radial and azimutal components in Prandtl's case $u_{\mathrm{r}}$ and $v_{\varphi}$, we must have, for $\varphi=\pi-\beta_{1}$, the conditions $u_{\mathrm{r}}=w_{2} \cos \beta_{1}, v_{\varphi}=w_{2} \sin \beta_{1}$. With the help of (3) and (21) this leads to a determination of $\varphi_{0}$ and $C$ by the following conditions

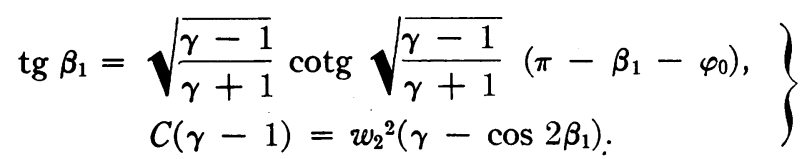

From the line $O B$ on, the path of the particles will be curved. The direction of velocity will bend round until it becomes parallel to the second plane $O E$ of the corner. This will occur in a second straight line $O D$ whose angle of inclination is given by a relation analogous to (22)

$$
\operatorname{tg}\left(\beta_{2}+\omega_{2}\right)=\sqrt{\frac{\gamma-1}{\gamma+1}} \operatorname{cotg} \sqrt{\frac{\gamma-1}{\gamma+1}}\left(\pi-\beta_{2}-\varphi_{0}\right) .
$$


Because of relation (20), the line $O D$ will be inclined under Mach's angle to the plane $O E$. Beyond $O D$ we have another region of parallel flow with velocity $w_{3}$ determined by $u_{\mathrm{r}}=w_{3} \cos \beta_{2}, v_{\varphi}=w_{3} \sin \beta_{2}$, for $\varphi=\pi-\beta_{2}$. The pressure in this region is constant and the same as in the line $\varphi=\pi-\beta_{2}$, which can be obtained from (11) and (21).

However, the type of flow around a corner, represented by Fig. 3 and described in the preceding paragraphs is not quite general. This becomes clear when we write out the equation of a stream line in Prandtl's solution, which is easily obtained from expression (21) for the potential. This equation becomes

$$
\mathrm{r}^{\frac{\gamma-1}{\gamma+1}} \cos \sqrt{\frac{\gamma-1}{\gamma+1}}\left(\varphi-\varphi_{0}\right)=C^{\prime}
$$

it represents a loop with its nearest point to the origin in $\varphi=\varphi_{0}$, going into infinity in the directions $\varphi-\varphi_{0}= \pm \sqrt{\frac{\gamma+1}{\gamma-1}} \frac{\pi}{2}$. (If we take $\gamma=1.4$,

this angle is $\pm 220^{\circ} 27^{\prime}$.) We see, therefore, that the angle by which the velocity can be turned is limited. Under certain conditions we may have a type of motion different from that of Fig. 3. As in that case, we shall have parallel flow up to the line $O B$ and Prandtl's solution beyond it. However, the bend of the velocities may reach its limit before they become parallel to the plane $O E$. In this case, the stream will go off under an angle to $O E$ leaving a wedge like sector of dead space between itself and this plane. This wedge will have pressure and density equal to zero: it will be pumped free of air by the suction of the current. It is not hard to see that the condition for this type of motion is

$$
\omega_{2}>\sqrt{\frac{\gamma+1}{\gamma-1}} \operatorname{arctg}\left(\sqrt{\frac{\gamma+1}{\gamma-1}} \operatorname{tg} \beta_{1}\right)-\beta_{1} .
$$

7. Flow Around a Quadrangle.-The formulae developed in sections $3,5,6$ suffice to give an answer to our problem: the resistance of a quadrangular contour. The original current (Fig. 1) with the velocity $w_{1}$ is limited by the shock wave and is converted into a flow of the velocity $w_{2}$ parallel to the side $A B$. This velocity and the pressure $p_{2}$ on the plane $A B$ can be computed by means of the formulae of section 4 . These data determine also the position of the line $B E$ which represents the limit of this type of motion. In the wedgelike space between $B E$ and $B F$ applies the Prandtl-Meyer solution. Our expressions of section 6 permit us to determine the position of the line $B F$ and the velocity and pressure obtaining in this line. This pressure $p_{3}$ is the same as that which prevails in the whole region of parallel flow beyond $B F$ and which is applied 
to the back plane $B C$. Finally, the flow encounters a second shock wave $C G$, but since we already possess all the data relating to the question of resistance, we need not discuss this last process in detail.

It is obvious that the resistance per unit length of our prism of section $A B C B^{\prime} A$ is given by the expression

$$
F=\left(p_{2}-p_{1}\right) S,
$$

where $S^{*}$ is the length of the diagonal $B B^{\prime}$ which we shall call the cross section of the projectile.

We compute the resistance for some typical cases. Let us take as initial velocity $w_{1}=3.36 a_{1}$, i.e., 3.36 times the velocity of sound in a medium having $\gamma=1.40$ and the pressure $p_{1}$. (For instance, in air under normal conditions: $\left.a_{1}=330 \mathrm{~m} / \mathrm{sec}, w_{1}=1110 \mathrm{~m} / \mathrm{sec}\right)$. The results can be summarized in the following table.

\begin{tabular}{|c|c|c|c|c|c|c|}
\hline $2 \omega t$ & $2 \omega_{2}$ & $p_{2}$ & $s_{3}$ & $F / S$ & $\zeta_{1} w_{1}^{2} \sin ^{2} \omega_{j}$ & $\rho_{2} / \rho_{1}$ \\
\hline $60^{\circ}$ & $16^{\circ} 40^{\prime}$ & 8.00 & 0.86 & 7.14 & \multirow{2}{*}{3.95} & 350 \\
\hline $60^{\circ}$ & $60^{\circ}$ & 8.00 & 0.14 & 7.86 & & 0.00 \\
\hline $16^{\circ} 40^{\prime}$ & $16^{\circ} 40^{\prime}$ & 1.95 & 0.74 & 1.21 & \multirow{2}{*}{0.33} & 0 \\
\hline $16^{\circ} 40^{\prime}$ & $60^{\circ}$ & 1.95 & 0.07 & 1.88 & & 1.00 \\
\hline
\end{tabular}

Pressures and $F / S$ are expressed in units $p_{1}$ (atmospheres). We see that the resistance has its highest value when the projectile is blunt from both ends, it is lowest when it is sharp from both ends. If the rear end is very abrupt, $p_{3}$ vanishes altogether.

As a second example, let us take a projectile with a front angle of $2 \omega_{1}=$ $29^{\circ}$ and an abrupt rear end. As we shall see in section 10 , we can interpose between the front part and the rear part any length of a parallepiped, parallel to the direction of motion, without changing the resistance. The computations are quite easy in this case, because the pressure on the back planes vanishes and we need only apply one formula, viz., equation (17) of section 4. This formula gives us the compression $x$ for every velocity $w_{1}$, while the pressure $F / S=p_{2}$ follows from formula (15).

$\begin{array}{lccccccccc}w_{1} \text { in } \mathrm{m} / \mathrm{sec} & 580 & 600 & 700 & 800 & 900 & 1000 & 1100 & 1200 & 1500 \\ F / S & 2.08 & 2.10 & 2.18 & 2.35 & 2.54 & 2.76 & 2.99 & 3.24 & 4.10 \\ F / S w_{1}^{2} & 6.20 & 5.84 & 4.45 & 3.68 & 3.13 & 2.76 & 2.43 & 2.24 & 1.83 \\ & & & & & & & & \\ w_{1} \text { in } \mathrm{km} / \mathrm{sec} & 2.0 & 2.5 & 3.1 & 4.0 & 5.26 & 9.0 & 13.1 & 34.7 \\ F / S & 5.79 & 8.10 & 11.5 & 17.3 & 29.0 & 81.5 & 169 & 1160 \\ F / S w_{1}{ }^{2} & 1.44 & 1.29 & 1.19 & 1.08 & 1.05 & 1.01 & 0.99 & 0.96\end{array}$

The theoretical limit for the ratio $F / S w_{1}^{2}$ is 0.94 . It should be pointed out that the current flows along the back surface only when $w_{1}$ is quite low. For higher velocities it detaches itself and forms a wedge of dead space at the rear side of the projectile. According to equation (24) the 
velocity at which the wedge extends into infinity is $6790 \mathrm{~m} / \mathrm{sec}$. For higher values of $w_{1}$ the currents, coming from the upper and the lower side, diverge leaving between them an open sector of dead space. As this dead space has the pressure zero, the result seems paradoxical: the question arises how it is possible to evacuate an infinite portion of space. This difficulty will be resolved in section 10 .

8. Discussion of Results.-An outstanding characteristic of our formulae is that they apply only to velocities $w_{1}$ above a certain lower limit. This appears, clearly, from expressions (9) and (17): for every given angle there exists a certain minimum value of $x$ below which the condensation cannot fall without making the square root of these expressions imaginary. This value turns out to be $x_{\text {min. }}=(1+\sin \omega)^{2} / \cos ^{2} \omega$, the corresponding value of $\alpha$ is $\alpha=45^{\circ}+\omega / 2$. The minimum velocity is then obtained from equation (17). For instance, we have ${ }^{6}$

$\begin{array}{cccc}\omega & x_{\min .} & \alpha_{\max } & { }^{2 v} \min . \\ 8^{\circ} 20^{\prime} & 1.339 & 49^{\circ} 10^{\prime} & 520 \mathrm{~m} / \mathrm{sec} \\ 14^{\circ} 30^{\prime} & 1.668 & 52^{\circ} 15^{\prime} & 580 \mathrm{~m} / \mathrm{sec} \\ 30^{\circ} & 3.000 & 60^{\circ} & 760 \mathrm{~m} / \mathrm{sec} \\ 45^{\circ} & 5.829 & 67^{\circ} 30^{\prime} & 1090 \mathrm{~m} / \mathrm{sec}\end{array}$

As the front angle increases the minimum velocity becomes higher and higher. This means that the type of motion, represented in Fig. 1 and characterized by a shock wave consisting of two separate branches $A D$ and $A D^{\prime}$ can develop only when the velocity $w_{1}$ is above this limit. For velocities below $w_{\min }$. and above the velocity of sound we should expect a front discontinuity of a different type (probably a single branched curved line not passing through the point $A$ ). If we plot the resistance against the velocity, we must distinguish three different parts of the curve: For velocities under that of sound $\left(w_{1}<a_{1}\right)$ we have a type of flow around the projectile without any discontinuities and, presumably, not very different from the potential flow. For velocities between $a_{1}$ and $w_{\text {min. }}$ a type of motion with a discontinuity of an unknown form. For $w_{1}>w_{\text {min. }}$ the conditions which we have analyzed in the preceding sections. These conclusions, though derived from considering a two dimensional model are completely borne out by observations with actual three dimensional projectiles. E.g., in $\mathrm{Cranz}^{\prime}$ book $^{7}$ we find on pp. 64 and 65 curves referring to bullets with sharp points. The quantity $F / S w_{1}^{2}$, is here plotted against the velocity $w_{1}$ and the three regions are very conspicuous. For $w_{1}<a_{1}$, the curve is almost horizontal, then we have a narrow region of a steep rise to a maximum which lies between 500 and $600 \mathrm{~m} / \mathrm{sec}$, corresponding to our $w_{\min }$, and beyond this a monotonic and slow decline. Our result, that $w_{\text {min. increases with the front angle, is }}$ also confirmed: on p. 62 some data for cylindrical projectiles (with flat 
front) are given and the maximum of the curve is not reached within the velocities of experimentation $(1300 \mathrm{~m} / \mathrm{sec})$.

Of the ballistical formulas for resistance proposed heretofore, those given by Sommerfeld ${ }^{8}$ and by $\mathrm{H}$. Loren $\mathrm{z}^{9}$ are partially based on theoretical considerations. The fundamental difference between these laws and ours is that they attempt to describe the -resistance for any velocity $w_{1}$ by a single formula. On the contrary, we maintain that in the three regions of velocity $w_{1}<a_{1}, a_{1}<w_{1}<w_{\text {min., }}, w_{1}>w_{\text {min. }}$, we must have different analytical expressions, since we have in them different types of hydrodynamical flow. Our solution applies only to the last region, and here it represents the observations in a satisfactory way. On the one hand, our formula which does not contain any empirical constants gives the right order of magnitude for the resistance. Our table at the end of section 7 shows that, for velocities of about $1000 \mathrm{~m} / \mathrm{sec}$, the resistance is about 2.8 $\mathrm{atm} / \mathrm{cm}^{2}$. Cranz and Becker (loc. cit., p. 63) measured for rifle bullets at the same velocity $1.8 \mathrm{~atm} / \mathrm{cm}^{2}$. On the other hand, the qualitative trend of our theoretical curve is the same as that of the curve given by Cranz. The quantity $F / S w_{1}^{2}$ decreases and approaches asymptotically a constant value. That the decay in our table (section 7 ) is somewhat slower than in Cranz' curves, is without significance: the resistance depends in our theory on the front angle $2 \omega_{1}$, and our choice of $29^{\circ}$, as an illustration, is purely accidental. The only discrepancy is the fact that our theoretical curve for $F / S$ starts with a horizontal tangent for $w_{1}=w_{\min }$. and then bends upward, while the experimental curve has an upward trend also in the beginning of the region. This may be a characteristic property of our two-dimensional model which, of course, cannot give a quantitative agreement with observations upon three dimensional projectiles.

It is here the place to return to the question of the existence of a second solution, touched upon in section 3 , and to explain why we did not attribute to this solution a physical reality. As we see from equation (9) the two solutions differ by the sign of the square root. For $x_{\min }$. (and $w_{\text {min. }}$ ) the square root vanishes and the two solutions coincide. We have pointed out that the angle $\alpha$ between the shock wave and the velocity $w_{1}$ has then the value $\alpha=45^{\circ}+\omega / 2$. As the compression $x$ increases, $\alpha$ becomes smaller in the first solution and larger in the second, the extreme values, for very large $x$, being $\alpha=\omega$ and $\alpha=\pi / 2$, respectively. The velocity $w_{2}$ in region (2) of figure 2 is given by $w_{2}=w_{1} \cos \alpha / \cos (\alpha-\omega)$. For a given $w_{1}$, it is smaller in the second solution than in the first. In fact, in the second solution, $w_{2}$ drops to values under the velocity of sound, for quite moderate velocities $w_{1}$, and continuous decreasing as $w_{1}$ goes up. This solution is, therefore, characterized by a higher potential energy of compression and by a lower kinetic energy than the first solution 
for the same $w_{1}$. Consequently, also the Lagrangean function and the principal function will be higher in it. According to Hamilton's principle every dynamical state tends to a minimum of the principal function, so that we conclude that the second solution is unstable and we have to deal only with the first. ${ }^{10}$

9. The Results Do Not Materially Depend on Special Assumptions.The formulas of the preceding sections are based on the assumption that the compression and expansion of air in its wave motion takes place without loss of energy by conduction and radiation. We may expect that this assumption breaks down when the temperature in parts of our wave becomes high, i.e., when the density $\rho_{2}$ and the velocity $w_{1}$ are large. Under these conditions the resistance depends almost exclusively upon the shock wave. The main question that must be answered is, therefore: how does the pressure $p_{2}$, in the shock wave, change, if we have deviations from the law (15) connecting pressure and density? It can be readily seen from equations (9) and (10) that these changes are very small, indeed. In the limiting case of extremely high compressions $\left(\rho_{2}>>\rho_{1}, x>>1\right)$, these equations give $\alpha=\omega$

$$
p_{2}-p_{1}=\rho_{1} w_{1}^{2} \sin ^{2} \omega \text {. }
$$

If the compression is fairly large and $\omega$ not over $45^{\circ}$, this expression gives an approximation. It is entirely independent of the law of compression, so that this law affects only small correetion terms. The writer took the trouble of computing the resistance, for the whole range of velocities $w_{1}$, using the adiabatic law instead of formula (15). Though the compression $x$ corresponding to every velocity $w_{1}$ is widely different in the two cases, the relation between $F / S$ and $w_{1}$ is, practically, the same. This is the reason why the adiabatic law could be used with comparative impunity.

The temperature $T_{2}$ in the shock wave can be obtained from equation (16) and goes up to rather high values:

$\begin{array}{lccccccc}w_{1} \text { in km/sec } & 1 & 2 & 3 & 5 & 9 & 19 & 35 \\ T_{2} \text { (Cent.) } & 104^{\circ} & 245^{\circ} & 500^{\circ} & 1300^{\circ} & 3700^{\circ} & 15.600^{\circ} & 53.000^{\circ}\end{array}$

The energy losses by conduction and radiation must be, therefore, considerable. In fact, $T_{2}$ is asymptotically proportional to $w_{1}^{2}$, while the time of contact with the projectile is inversely proportional to the first power of $w_{1}$. The relative importance of conduction increases as $w_{1}$, that of radiation at a much faster rate. Although this fact does not materially affect the law of resistance, it is interesting to discuss its implications. Formula (17) seems to indicate that there is an absolute maximum for $x$ and for $\omega$. In fact, when $x=(\gamma+1) /(\gamma-1), w_{1}=\infty$; on the other hand, when $\operatorname{tg} \omega>1 / \sqrt{\gamma^{2}-1}$, the square root is imaginary 
for all permissible values of $x$. But owing to conduction and radiation these limitations have no physical reality. The real temperature will be much lower than that computed from formula (16) and, therefore, the compression $x$ much higher (since $p_{2}$ remains, practically, unchanged). Because of this, our theory will be applicable to any front angle up to the neighborhood of $90^{\circ}$, and formula (26) will represent a very close approximation of the real pressure at high velocities.

The thermic energy losses will affect also the character of the Prandt1Meyer expansion waves. Here, too, we must expect, for very high velocity $w_{1}$, much smaller differences of temperature and pressure than would follow from the adiabatic law. In the case of isothermic expansion, we should have, instead of equations (14)

$$
\begin{gathered}
\psi=\sqrt{R T} r\left(\varphi-\varphi_{0}\right), \\
\log \rho=\frac{C}{R T}-1-\frac{\left(\varphi-\varphi_{0}\right)^{2}}{2} .
\end{gathered}
$$

The equation of a stream line would take the form

$$
r=C^{\prime} \exp \frac{1}{2}\left(\varphi-\varphi_{0}\right)^{2} .
$$

This equation shows that there is no limiting angle for the deflection as in the case of the adiabatic law. The stream lines around a convex angle, under all circumstances, will be parallel to the sides and a dead space will never be formed.

With increasing velocity $w_{1}$, the conduction and radiation begin to dominate the heat exchange and the actual flow begins to resemble these conditions closer and closer. The dead space becomes narrower and narrower and in the limit $\left(w_{1}=\infty\right)$ the flow forms only a thin film on the surface of the polygon.

10. Generalizations and Limiting Values.-In the preceding sections we have computed the resistance for quadrangles. The same method leads itself to the treatment of convex polygonal contours which may be chosen symmetrical or asymmetrical with respect to the direction of motion. We have, in this case, shock waves, in the front and in the rear, and Prandtl wedges at all the remaining corners. It is obvious, from the character of Prandtl's solution, that the pressure on any side of the polygon depends only on the two angles which this side and the front side include with the direction of motion. It is independent of the position and length of all the intermediate sides of the polygon. This circumstance permits the use of our formulas of sections 4 and 5 for computing the pressures on all sides of the polygon: we have only to take as $\omega_{2}$ the angle between the front side and the side we are considering. We can say, therefore, that the solution for a polygon with any number of sides is explicitly contained in our formulas. 
However, in extending the method to polygonal contours, one should restrict himself to cases when none of the angles $\omega_{2}$ do satisfy the inequality (24). This precaution is necessary for the following reason: The lines $A B$ and $B E$ of figure 1 intersect, if continued. The region of flow parallel to $A B$ is limited to a finite triangle. Though we have no accurate expression for the state of motion above this triangle, it is safe to say that the influence of the projectile will not extend far beyond the point of intersection. At a certain distance beyond this point we shall find again the original parallel flow, as it exists to the left of the point $A$. If our contour is such that we have, in addition to $A B$, other sides sticking out above the point $B$, it may happen that one of these sides will be also above the point of intersection of $A B$ and $D E$ and will receive its pressure from a portion of the fluid which has not passed through the shock wave $A B$. While restricting the application of the method, this consideration removes the difficulty mentioned at the end of section 6 . The state of motion analyzed in the preceding sections is restricted to a strip of finite width behind the projectile and, consequently, the energy necessary to maintain the motion is also finite.

Of particular interest to the writer is the limiting case of very high velocities $w_{1}$. We have seen in the preceding section, that in this case the compression $x$ is very large and that the angle $\alpha$ approaches the limiting value $\alpha=\omega_{1}$. Neglecting in formula (26) $p_{1}$ beside $p_{2}$ we obtain the law of resistance

$$
F / S=\rho_{1} w_{1}^{2} \sin ^{2} \omega_{1}
$$

This law can be considerably generalized. What will happen, if we have a polygon instead of the quadrangle of Fig. 1? With increasing velocity $w_{1}$ the intersection of $A D$ and $B E$ comes nearer and nearer to the point $B$, and in the limit $w_{1}=\infty$, it strictly coincides with it. The whole triangle is reduced to an infinitesimal film covering the side $A B$. We have seen, also, that at the same time the current turns completely in the wedge adjacent to $B E$ and becomes parallel to the next side. It follows that this side, which we suppose to be sticking out beyond $A B$ is completely exposed to the original parallel flow, exactly like the side $A B$ itself. Consequently, the pressure on this side can be computed by the same formula (27). The resistance of a polygon is, therefore, given by the formula

$$
F / S=\rho_{1} w_{1}^{2} \overline{\sin ^{2} \omega} .
$$

where $\overline{\sin ^{2} \omega}$ is the mean squared sine of the angles which the different sides of the polygon include with the velocity $w_{1}$. The average must be taken only over sides turned toward the current (front half of the polygon), 
as the pressure on the back half vanishes. Since a closed curve can be regarded as the limiting case of a polygon, expression (28) applies also to contours limited by a continuous curve. Finally, it is easy to see that the limiting value (28) will be correct also for three dimensional convex bodies. Though our theory gives no quantitative description of the three dimensional case, qualitatively the phenomenon is the same in both cases. Especially it will be true, also in the three dimensional case, that in the limit of very high velocities the surface of discontinuity (shock wave) will closely envelop the surface of the body and the region of compression will form only a thin film on this surface. Since every small part of a thin film can be considered as plane, we can apply to it the formulas developed for the two dimensional case and arrive, in this way, at expression (28).

To avoid misunderstandings it should be emphasized that, in the preceding paragraphs, a velocity is considered "high," if it is capable of causing a large compression $x$. The point, where low velocities end and high velocities begin, depends entirely on the compressibility of the medium.

${ }^{1}$ B. Riemann, Gesammelte Werke, p. 156 . Also: Riemann-Weber, Partielle Differentialgleichungen, Vol. II., pp. 469-498. Braunschweig 1901.

${ }^{2}$ L. Prandtl, Phys. Zs., 5, p. 599 (1904).

${ }^{3}$ Th. Meyer, Mitteilungen des Vereins deutscher Ingenieure. Berlin 1908.

${ }^{4}$ E. Mach, Wien. Ber., 77, p. 7; p. 819 (1878).

${ }^{5}$ This reasoning is accurate only then when the velocity of sound is the same in all directions.

${ }^{6}$ For large front angles $\left(30^{\circ}\right.$ and $\left.45^{\circ}\right)$ and low velocities (vicinity of $\left.w_{\min }\right), w_{2}$ comes out smaller than the velocity of sound $a_{2}$. This result may have no physical reality because of thermal conduction: compare section 9 .

7 C. Cranz, Äussere Ballistik. Vol. I. Berlin 1925.

${ }^{8}$ F. Klein and A. Sommerfeld, "Theorie des Kreisels." Bd. IV, Chapter "Ballistik." Leipzig, 1910.

${ }^{9} \mathrm{H}$. Lorenz, Zs. Vereins deutscher Ingenieure, p. 625 (1916).

${ }^{10}$ This argument is not rigorous, but it is possible to give a rigorous proof. 